\title{
Expectations of barriers to psychosocial care: views of parents and adolescents in the community
}

\author{
Marieke Nanninga $^{1} \cdot$ Sijmen A. Reijneveld $^{1}$ Erik J. Knorth ${ }^{3}$. \\ Danielle E. M. C. Jansen ${ }^{1,2}$
}

Received: 29 January 2015 / Accepted: 22 April 2015 / Published online: 13 May 2015

(C) The Author(s) 2015. This article is published with open access at Springerlink.com

\begin{abstract}
Parents with a child suffering from psychosocial problems frequently experience barriers to psychosocial care, which may hinder access. Expectations of barriers may have the same effect, but evidence is lacking. The aim of this study is to examine parents' and adolescents' expectations of barriers regarding psychosocial care for the child, along with associated child and family characteristics. We obtained data on an age-stratified random sample of school children/pupils aged 4-18 via questionnaires $(N=666$; response rate $70.3 \%)$. Expectations of barriers to psychosocial care were measured with the "Barriers to Treatment Participation Scale-Expectancies" questionnaire (BTPS-exp). Results showed that $64 \%$ of the parents of children below age $12,59 \%$ of the parents of adolescents (age 12-18), and $84 \%$ of the adolescents expected one or more barriers. Parents and adolescents expected barriers most frequently with respect to irrelevance of treatment. Mainly parents with low educational level and their adolescents expected barriers regarding treatment, and quite a few characteristics of parents of adolescents were associated with expecting multiple barriers regarding treatment demands and issues, for example, single parents, parents of lower educational level and of adolescent boys, and parents of adolescents with psychosocial problems. We conclude
\end{abstract}

Marieke Nanninga

m.nanninga02@umcg.nl

1 Department of Health Sciences, University Medical Center Groningen, University of Groningen, Antonius Deusinglaan 1/FA10, 9713 AV Groningen, The Netherlands

2 Department of Sociology and Interuniversity Center for Social Science Theory and Methodology (ICS), University of Groningen, Groningen, The Netherlands

3 Department of Special Needs Education and Youth Care, University of Groningen, Groningen, The Netherlands that adolescents especially, but also their parents and parents of younger children, expect major barriers to psychosocial care, which may greatly hinder appropriate care seeking. This evidence may support professionals and policymakers in their attempts to improve access to psychosocial care.

Keywords Child · Adolescent - Psychosocial care · Barriers to care $\cdot$ Health services accessibility

\section{Introduction}

Only a minority of the children and adolescents with emotional or behavioral problems receive psychosocial care [1-12]. For example, a recent study showed that, of the adolescents in need of psychosocial care, only $29 \%$ actually received that care [11]. One of the causes may be the barriers to care that parents and children expect or have experienced [13, 14]. According to Owens and colleagues, barriers to care comprise those "factors that have prevented access or created difficulties in accessing child mental health services" (p.731) [15]. Between 35 and $61 \%$ of parents with a child suffering from psychosocial problems experience barriers to care $[15,16]$. These barriers can be structural-financial, for example — but can also relate to perceptions of care or psychosocial problems: the prospect of encountering inadequate care providers or the expectation that problems will improve by themselves, for example $[15,17]$.

Most evidence about barriers to care is based on clinical samples, i.e., children and adolescents with psychosocial problems either in need of or using psychosocial care [15-21]. This evidence shows that the barriers experienced by those in need have been shown to be associated 
with a lower intention of seeking help [18], along with a lower likelihood of using psychosocial care [19-21]. Barriers experienced by parents during their child's treatment have been shown to be associated with poor care outcomes: higher dropout rates, less symptom improvement, and less treatment acceptance by the child and parent [17, 22, 23].

To our knowledge, community-based evidence is lacking in terms of expectations that children, adolescents, and parents have about barriers to children's psychosocial care. These expectations are likely to influence care seeking and use. For example, according to Andersen's Health Behaviour Model, expectations regarding health care are among the determinants for patients' utilization of care [24-26]. Furthermore, the review of Morrisey-Kane and Prinz concluded that positive parental expectations towards care are of importance for successful help seeking and engagement in treatment [14].

The aim of our study was to examine in a community sample of children and adolescents: (1) the number of barriers that parents and adolescents expect when considering seeking psychosocial care for their child or for themselves, respectively; (2) the type of barriers expected most frequently; and (3) the child and family characteristics associated with these expectations. In this study, psychosocial care is defined as all care aimed at reducing or making manageable psychosocial problems of children and adolescents [27]. In the Netherlands, like in many other countries, this is provided by preventive child health care, child and adolescent social care, and mental health care [28-30]. Knowledge about the expectations of parents and adolescents regarding barriers to care may provide direction to improve the help-seeking and treatment process.

\section{Methods}

\section{Study design}

We used data from the first measurement wave of the community sample of a large prospective cohort study called TakeCare [28, 31]. TakeCare is conducted by the Collaborative Centre on Care for Children and Youth (C4Youth) and is designed to investigate the trajectories and outcomes of children aged 4-18 receiving psychosocial care in one Dutch region. The design was assessed by the Medical Ethical Committee of the University Medical Center Groningen, and approved without needing full assessment. Informed consent was obtained from all participating respondents.

\section{Sample and procedure}

We used a stratified random sample of school children and pupils $(N=1025)$, obtained via five primary schools, two secondary schools, and one school for intermediate vocational education, recruited by taking into account the distribution of children and adolescents across the study region according to their age, gender, socioeconomic position, and degree of urbanization. Parents/caregivers of children aged $4-18$ years old and adolescents (age $\geq 12$ ) were invited to participate between April 2011 and June 2013. Children with insufficient understanding of Dutch, living outside the northern region, or following special education because of intellectual disability were excluded $(N=77)$. Of the eligible 948 respondents, 666 participated, i.e., either the child and/or the parent (response $70.3 \%)$. The main reasons for non-participation were opting out $(N=99)$. Differences between respondents and non-respondents were small for age, gender, degree of urbanization, and severity of psychosocial problems (the latter based on one impact-question of the Strengths and Difficulties Questionnaire (SDQ) [32]), with Cohen's effect sizes ranging from 0.02 (psychosocial problems) to 0.08 (degree of urbanization). Differences between respondents and children in the community were small for age and gender, with effect sizes being 0.00 and 0.01 , respectively [28].

Data were obtained from parents/caregivers and adolescents via web-based or paper questionnaires, and, if needed, we provided assistance in filling out the questionnaire. Participants were frequently reminded about filling out the questionnaire, and returned questionnaires were checked for completeness to reduce the chance of missing data. Participants were rewarded with a gift card worth ten euros.

\section{Measures}

Parents' and adolescents' expectations of barriers to care were measured using the Barriers to Treatment Participation Scale-Expectancies (BTPS-exp) [17, 33], translated into Dutch following the Guillemin translation procedure [34]. Parents and adolescents were asked to "imagine that you are seeking psychological help, counseling, or advice [for your child]" and asked to indicate to what extent they agreed with the items, with answer categories ranging from "totally disagree" to "totally agree" (5-point Likert scale; 44 items in the parent version, 43 items in the adolescent version). An expected barrier was coded as occurring when rated with "somewhat agree" or "totally agree" to calculate the total number of expected barriers. In addition, we calculated mean scores for the total scale and for each of the subscales:

- Stressors and obstacles competing with treatment, i.e., problems regarding transport, other children at home, activities, health, or conflict with a significant other 
about coming to treatment (parent and adolescent versions 20 and 19 items, respectively)

- Treatment demands and issues, i.e., concerns about treatment cost and duration, having a voice in treatment, confusing information (10 items)

- Perceived irrelevance of treatment, i.e., concerns about the need for and relevance of treatment, about treatment introducing new or other problems (8 items)

- Problematic relationship with therapist, i.e., concerns about not having a good relationship with the therapist, not receiving enough support (6 items).

Internal consistencies of the total scale and subscales of the parent and adolescent versions were good (lowest Cronbach $\alpha=0.83$ ).

\section{Child characteristics}

Relevant child characteristics included age, gender, ethnicity, psychosocial problems, and past psychosocial care use. Ethnicity was defined as either Dutch or non-Dutch (i.e., the child and/or one of the parents was foreignborn). Children's psychosocial problems were measured using the total difficulties score of the "Strengths and Difficulties Questionnaire" (SDQ-TDS), based on the past 6 months (Cronbach's $\alpha$ parent version $=0.80$; adolescent version $=0.72$ ) $[31,35-37]$. The score consists of 20 items describing positive and negative attributes of children on the following dimensions: emotional symptoms, conduct problems, hyperactivity/inattention, and peer problems. The scale was dichotomized into the "normal" and the "borderline to abnormal" range of the SDQ.

Psychosocial care use in past 6 months was measured using the "Questionnaire Intensive Care for Youth" [3840]. We asked parents and adolescents to indicate among a list of professionals and types of care whether they had contact because of psychosocial problems. A child using psychosocial care was defined as one whose parent (or the adolescent him/herself) indicated that they or the child had contacted a professional (i.e., a general practitioner, psychologist, or psychiatrist) and/or used care (i.e., outpatient social care, day or residential treatment, foster care) for psychosocial problems of the child in the past 6 months.

\section{Family characteristics}

The family characteristics included were parental educational level and family composition. Parental educational level was based on the highest educational level achieved by either one of the parents/caregivers [41]. The categories "primary education" and "lower levels of secondary education" were combined into one category, because only a few parents fell into the first category.

Family composition was assessed by asking the parent with whom the child lived. Answers were categorized as "two-parent family" or "other" (e.g., living with one parent, a foster family, or living in a residential care facility).

\section{Statistical analyses}

We first described the background characteristics of the sample, for parents of children (aged 4-12), parents of adolescents (aged 12-18), and adolescents separately. Next, we assessed their number of expectations regarding barriers to care (aim 1), and the types of barriers most frequently expected (aim 2). Differences between the scores of parents and adolescents were calculated using Pearson Chi-square tests and non-parametric Mann-Whitney $U$ tests because of the scales' skewed distributions.

We performed univariable logistic regression analyses to assess the crude associations of child and family characteristics with expecting multiple barriers of each type. In addition, we performed multivariable logistic regression analyses to assess the adjusted associations (aim 3). Both crude and adjusted analyses were performed with Generalized Estimating Equations modeling (GEE) (with exchangeable correlation structure) to account for possible inter-correlations between children from the same school. The BTPS-exp subscales were dichotomized as the $25 \%$ highest scores vs. lower scores (Table 1) because of the skewed distributions. For all logistic regression analyses, odds ratios (OR) and $95 \%$ confidence intervals (CI) were presented. A $p$ value $<0.05$ was considered statistically
Table 1 Range of the $25 \%$ highest scores for the subscales of the BTPS-exp

\begin{tabular}{|c|c|c|c|c|c|c|}
\hline \multirow[t]{2}{*}{ Score (possible scores range from 1 to 5 ) } & \multicolumn{2}{|c|}{$\begin{array}{l}\text { Parents } \\
\text { children }<12 \text { years }\end{array}$} & \multicolumn{2}{|c|}{$\begin{array}{l}\text { Parents } \\
\text { adolescents }\end{array}$} & \multicolumn{2}{|c|}{ Adolescents } \\
\hline & From & To & From & To & From & To \\
\hline Stressors and obstacles & 1.55 & 5.00 & 1.45 & 5.00 & 2.53 & 5.00 \\
\hline Demands and issues & 2.44 & 5.00 & 2.50 & 5.00 & 2.78 & 5.00 \\
\hline Perceived irrelevance & 3.00 & 5.00 & 3.00 & 5.00 & 3.29 & 5.00 \\
\hline Problematic relationship with therapist & 2.60 & 5.00 & 3.00 & 5.00 & 3.00 & 5.00 \\
\hline
\end{tabular}


significant (two-sided test). Analyses were performed using SPSS Statistics version 20.

\section{Results}

\section{Sample characteristics and barrier expectations}

Based on parent reports, $10.9 \%$ of the children and $10.8 \%$ of the adolescents had psychosocial problems, and $26.9 \%$ and $30.2 \%$ of all children and adolescents had used psychosocial care in the past 6 months, respectively. Of the adolescents, $21.1 \%$ reported psychosocial problems, and $34.6 \%$ reported use of psychosocial care in the past 6 months (Table 2). The majority of the parents expected one or more barriers, i.e., 63.6 and $58.9 \%$ of the parents of children and adolescents, respectively. Of the adolescents, $83.9 \%$ expected one or more barriers (Table 3).

Regarding the type of barrier expectations, both parents and adolescents expected barriers of the type "perceived irrelevance of treatment" most frequently, followed by "problematic relationship with therapist," "treatment demands and issues," and, lastly, "stressors and obstacles competing with treatment" (Table 3).

Overall, no significant differences were found between the scores of parents of children and parents of adolescents, except for the subscale "problematic relationship with therapist." Scores of adolescents were statistically significantly higher than scores of their parents, except for scores on the subscale "problematic relationship with therapist."
Table 2 Child and family characteristics of the participants

\begin{tabular}{|c|c|c|c|c|c|c|}
\hline \multirow[t]{3}{*}{ Characteristics } & \multirow{2}{*}{\multicolumn{2}{|c|}{$\begin{array}{l}\text { Parents } \\
\text { children }<12 \text { years }\end{array}$}} & \multirow{2}{*}{\multicolumn{2}{|c|}{$\begin{array}{l}\begin{array}{l}\text { Parents } \\
\text { adolescents }\end{array} \\
N=278^{\mathrm{b}}\end{array}$}} & \multirow{2}{*}{\multicolumn{2}{|c|}{$\begin{array}{l}\text { Adolescents } \\
N=280^{\mathrm{b}}\end{array}$}} \\
\hline & & & & & & \\
\hline & $N$ & $(\%)$ & $N$ & $(\%)$ & $N$ & $(\%)$ \\
\hline \multicolumn{7}{|l|}{ Child characteristics } \\
\hline \multicolumn{7}{|l|}{ Gender } \\
\hline Male & 163 & 44.3 & 127 & 45.7 & 116 & 41.4 \\
\hline Female & 205 & 55.7 & 151 & 54.3 & 164 & 58.6 \\
\hline \multicolumn{7}{|l|}{ Ethnicity } \\
\hline Dutch & 336 & 93.6 & 242 & 89.3 & 233 & 90.3 \\
\hline Non-Dutch & 23 & 6.4 & 29 & 10.7 & 25 & 9.7 \\
\hline \multicolumn{7}{|l|}{ Psychosocial problems } \\
\hline Normal & 328 & 89.1 & 248 & 89.2 & 221 & 78.9 \\
\hline Borderline & 15 & 4.1 & 12 & 4.3 & 33 & 11.8 \\
\hline Abnormal & 25 & 6.8 & 18 & 6.5 & 26 & 9.3 \\
\hline \multicolumn{7}{|l|}{ Psychosocial care use in past 6 months } \\
\hline No & 269 & 73.1 & 194 & 69.8 & 183 & 65.4 \\
\hline Yes & 99 & 26.9 & 84 & 30.2 & 97 & 34.6 \\
\hline \multicolumn{7}{|l|}{ Family characteristics } \\
\hline \multicolumn{7}{|l|}{ Parental educational level } \\
\hline Primary education & 2 & 0.5 & 1 & 0.4 & 1 & 0.4 \\
\hline Lower levels of secondary education & 21 & 5.7 & 31 & 11.2 & 25 & 9.7 \\
\hline Higher levels of secondary education & 189 & 51.5 & 126 & 45.7 & 119 & 46.1 \\
\hline Senior vocational education & 119 & 32.4 & 83 & 30.1 & 78 & 30.2 \\
\hline University & 36 & 9.8 & 35 & 12.7 & 35 & 13.6 \\
\hline \multicolumn{7}{|l|}{ Family composition } \\
\hline Biological two-parent family & 252 & 68.7 & 166 & 59.7 & 212 & 75.7 \\
\hline Other & 115 & 31.3 & 112 & 40.3 & 68 & 24.3 \\
\hline
\end{tabular}

a Numbers do not always add up to $N=368$ due to missing data

${ }^{\text {b }} N=258(86.0 \%)$ couples of parents and adolescents participated, $N=20(6.7 \%)$ parents participated without participation of the adolescent, and $N=22(7.3 \%)$ adolescents participated without participation of the parent 
Table 3 Parents' and adolescents' barrier expectations: frequencies and means

\begin{tabular}{|c|c|c|c|c|c|c|}
\hline \multirow[t]{2}{*}{ Expectations of barriers } & \multicolumn{2}{|c|}{$\begin{array}{l}\text { Parents } \\
\text { children }<12 \text { years }\end{array}$} & \multicolumn{2}{|c|}{$\begin{array}{l}\text { Parents } \\
\text { adolescents }\end{array}$} & \multicolumn{2}{|c|}{ Adolescents } \\
\hline & $N$ & $(\%)$ & $N$ & $(\%)$ & $N$ & $(\%)$ \\
\hline \multicolumn{7}{|l|}{ Number (range $1-44^{\mathrm{a}}$ ) } \\
\hline 0 & 133 & $(36.3)$ & 114 & $(41.0)$ & 45 & $(16.2)^{\mathrm{c}, * *}$ \\
\hline 1 & 88 & $(24.0)$ & 61 & $(21.9)$ & 31 & $(11.2)^{\mathrm{c}, * *}$ \\
\hline 2 & 44 & $(12.0)$ & 29 & $(10.4)$ & 25 & $(9.0)^{\mathrm{c}, * *}$ \\
\hline$\geq 3$ & 101 & $(27.6)$ & 74 & $(26.6)$ & 177 & $(63.7)^{\mathrm{c}, * *}$ \\
\hline Total number (M, SD) & 1.99 & $(2.9)$ & 2.32 & $(3.7)$ & 6.28 & $(6.6)^{\mathrm{c}, * *}$ \\
\hline Score (range 1-5) & $\mathrm{M}$ & $(\mathrm{SD})$ & $\mathrm{M}$ & (SD) & $\mathrm{M}$ & (SD) \\
\hline Barriers total & 1.63 & $(0.5)$ & 1.67 & $(0.6)$ & 2.11 & $(0.7)^{\mathrm{c}, * *}$ \\
\hline Stressors and obstacles & 1.35 & $(0.5)$ & 1.33 & $(0.5)^{\mathrm{b}, \#}$ & 1.91 & $(0.8)^{\mathrm{c}, * *}$ \\
\hline Demands and issues & 1.72 & $(0.8)$ & 1.76 & $(0.8)$ & 2.05 & $(0.8)^{\mathrm{c}, * *}$ \\
\hline Perceived irrelevance & 2.12 & $(0.8)$ & 2.23 & $(0.9)$ & 2.62 & $(0.9)^{\mathrm{c}, * *}$ \\
\hline Problematic relationship with therapist & 1.76 & $(0.8)$ & 1.95 & $(0.9)^{\mathrm{b}, *}$ & 2.09 & $(0.9)^{\mathrm{c}, \#}$ \\
\hline \multicolumn{7}{|l|}{$M$ mean, $S D$ standard deviation } \\
\hline \multicolumn{7}{|l|}{${ }^{\text {a }}$ Range $1-43$ for adolescents } \\
\hline \multicolumn{7}{|c|}{$\begin{array}{l}\text { b Significant differences between parents of children }<12 \text { years and parents of adolescents, }{ }^{\#}<0.10 \text {; } \\
* p<0.05\end{array}$} \\
\hline
\end{tabular}

\section{Association of child and family characteristics with barrier expectations}

The results of univariable logistic regression analyses showed that, among the three groups, there were a series of variables associated with expecting different types of multiple barriers. This was particularly true of "stressors and obstacles competing with treatment", "treatment demands and issues" and "problematic relationship with therapist" (Table 4).

The results of multivariable logistic regression analyses showed that expecting multiple barriers of the type "stressors and obstacles" was more likely in parents of children with a low educational level (Table 4). For parents of adolescents, this was more likely when their child was a boy, was of non-Dutch ethnicity or from other than two-parent families. For adolescents themselves, this was more likely for girls, when they had psychosocial problems, had used psychosocial care previously and when their parents had a university educational level (in contrast with a senior vocational education).

Expecting multiple barriers of the type "treatment demands and issues" was more likely for parents of children with a low parental educational level. For parents of adolescents, this was more likely for parents of adolescent boys, of adolescents with psychosocial problems, parents with higher levels of secondary education (compared to university level), and for parents in other than a biological two-parent family. For adolescents, this was more likely for boys, for those who had used psychosocial care previously and for those who had parents with a higher secondary educational level (compared to university level).

Expecting multiple barriers of the type "perceived irrelevance of treatment" was, in the adjusted models, not statistically significantly associated with any of the child and family characteristics for parents of children. For parents of adolescents, this type of expected barriers was more likely when they were of non-Dutch ethnicity or had low education. For adolescents, it was more likely with parents who had primary or lower levels of secondary education (compared to university) and for adolescents with parents who had university education compared to senior vocational education.

Lastly, when it came to expecting multiple barriers of the type "problematic relationship with therapist," none of the examined characteristics of parents of children were significantly associated. For parents of adolescents, expecting multiple barriers of this type was more likely in parents of boys, of non-Dutch ethnicity and with low and medium educational level; for adolescents this was more likely when they had psychosocial problems and when their parents had low and medium educational level.

\section{Discussion}

Our community-based study showed that the majority of parents and adolescents expected barriers to child and adolescent psychosocial care. Adolescents expected barriers more frequently than their parents did. The most frequently 


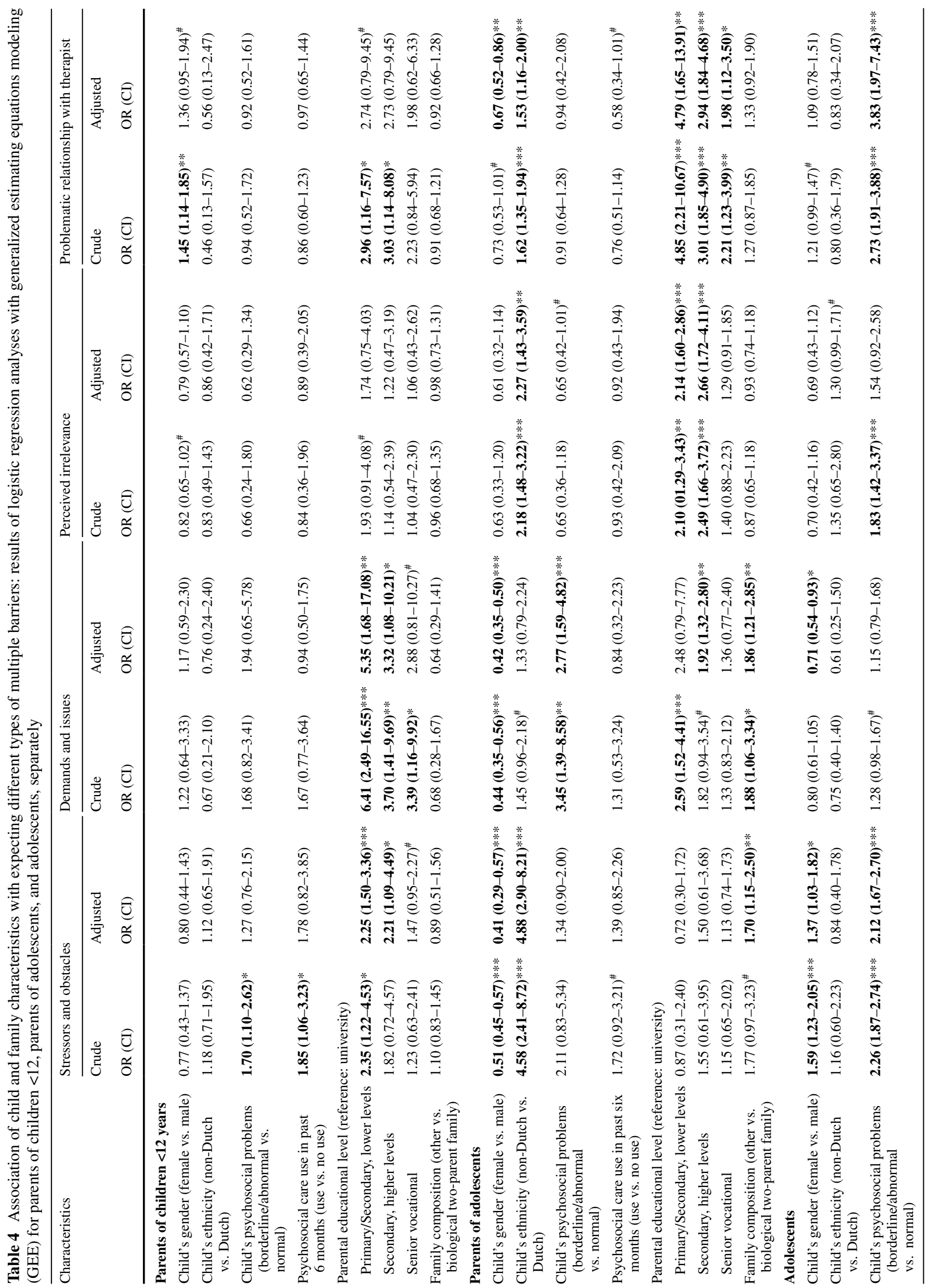




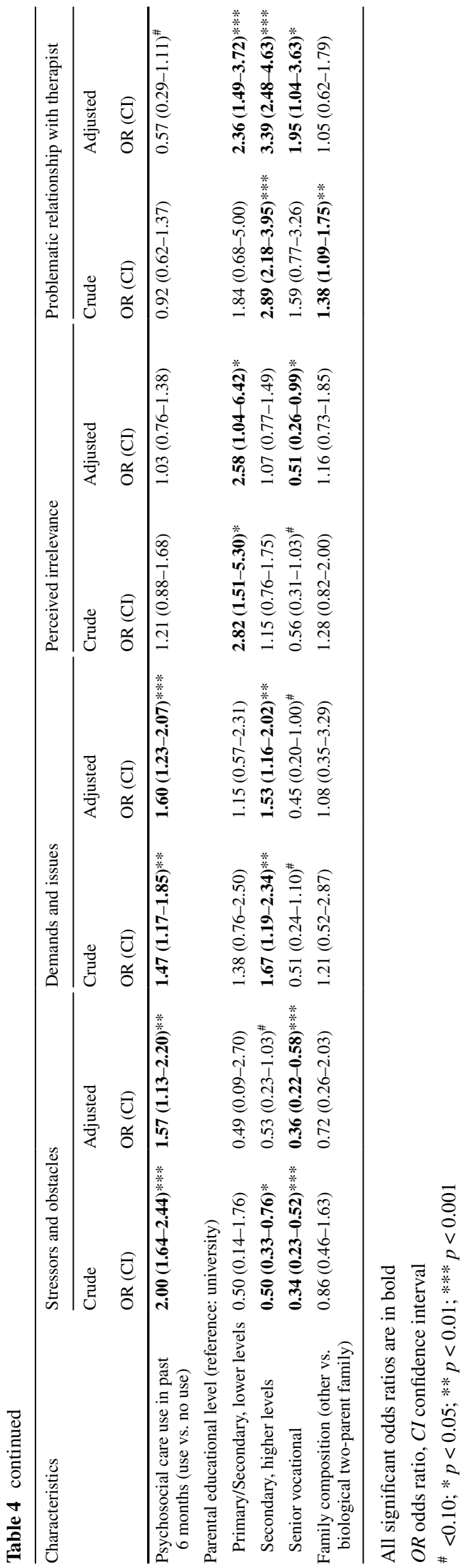

expected barriers were those with respect to treatment irrelevance, problematic relationship with therapists, and treatment demands. Several child and family characteristics were associated with almost all types of barriers, except for irrelevance of treatment. It was mainly parents with low educational level and their adolescents who expected barriers regarding treatment. Especially for parents of adolescents quite a few characteristics were associated with expecting multiple barriers regarding treatment demands and issues, e.g., single parents, with lower educational level, parents of male adolescents, and of adolescents with psychosocial problems.

\section{Interpretation and fit with other studies}

To our knowledge, this study is the first that assessed parents' and adolescents' expectations of barriers to child and adolescent psychosocial care. Findings on the number and types of expected barriers point in the same direction as the findings for studies on barriers experienced in accessing care, except for one study that showed a much smaller number of perceived barriers $[15,16]$. The latter might have been specific to a mainly African-American low-income sample, or might simply imply that expectations and actual experiences of barriers differ.

Our study showed that the number of children and adolescents who used psychosocial care in the last 6 months was higher than the number that reported having psychosocial problems. This seems to be in contrast with findings of studies so far $[3,11]$. It might suggest that psychosocial problems of children and adolescents are reduced due to successful treatment. However, information about causality is lacking because both problems and psychosocial care use refer to the same time period. The finding might also be explained by the fact that our definition of psychosocial care use is rather broad: we measured any care aimed at reducing or making manageable psychosocial problems of children and adolescents. For example, adolescents mainly use general care for psychosocial problems instead of specialized social or mental health care [29]. It is quite likely that (parents of) some children and adolescents consulted general care providers because of worries about psychosocial issues which are not necessarily scored as problems on the SDQ.

Regarding barriers, adolescents expected barriers more frequently than their parents did, a finding which implies that it is worthwhile to assess the expectations of both. This is consistent with evidence showing that parents and their children often differ in their views, including the well-known example of their views on psychosocial problems [42-44]. It might be that children in general foresee barriers more often than their parents do, because they are the ones who have to follow treatment and have to address 
their problems. This may be especially true for adolescents. Their developmental stage is characterized by a growth in autonomy, in self-directedness, and in the desire to solve problems on their own, which is brought about by creating distance from and less dependency on their parents and educators [45-47]. This may lead to an avoidance of interference from others, such as psychosocial care professionals, which might be reflected in the expectation of multiple barriers to care.

The most frequently expected type of barrier was the expectation that treatment would be irrelevant. For parents of children this result emerged regardless of sociodemographic background. This might reflect that these parents prefer to solve problems on their own or within their informal networks. It might also mean that there is a lack of confidence in the quality of the treatment and its effectiveness [47]. Parents of adolescents of non-Dutch ethnicity more often expected this type of barriers as well as barriers regarding the relationship with the therapist and stressors and obstacles at home. It might also be that parents from some cultures expect or have experienced resistance to arranging psychosocial care for the child more often, because of a greater stigma attached to psychosocial problems and to using psychosocial care [48-50].

Barriers regarding treatment-i.e., irrelevance, problematic relationship with therapists, and demands and issues-were more frequently expected by parents of low educational level and their adolescents. These families, as compared to families with higher educational level, more often lack the skills needed to navigate the mental health care or social care systems, which might explain our finding. These families more often have limited health literacy, for example, which is a factor that has been shown to be associated with distrust of professionals and pessimism about treatment [51].

Furthermore, barriers related to treatment demands and issues were expected by parents and adolescents who already (might) had some experience with seeking psychosocial care. That is by parents of adolescents with psychosocial problems and adolescents who had used psychosocial care in the past. Also, adolescents who had psychosocial problems expected barriers regarding a problematic relationship with a therapist. It might be that seeking or using psychosocial care is actually the current reality for them. Their expectations might well be based on actual experiences, which is something parents and adolescents do not have if they are just imagining having to seek psychosocial care [52]. This explanation might also hold for our other findings, for example on the association with parental educational level, non-Dutch ethnicity and one-parent families because these children also are more at risk and more often show psychosocial problems [12, 48, 53-55].
Barriers of single parents, i.e., regarding treatment demands and stressors at home, might, for example, be based on the burden they experience from having to organize the household on their own, or on a lack of finances to meet treatment costs. Parents of adolescent boys, who expected barriers regarding treatment demands and a problematic relationship with a therapist, might for example, more often be apt to expect that their child would refuse to attend treatment, simply as an expression of the more externalizing behavior pattern that boys show [56], as compared to parents of female adolescents. This might also be the reason why adolescent boys themselves expected these barriers.

Barriers related to stressors and obstacles that compete with treatment, which are mainly dependent on the family's home situation, were expected on the part of parents of adolescent boys, and of one-parent families, of adolescents with psychosocial problems and/or who had used psychosocial care in the past. In addition, these findings might be explained by previously experienced barriers. However, it remains unclear why adolescent girls mainly expect this type of barriers, whereas the association is the other way around for their parents.

Lastly, adolescents with parents with a university degree were especially likely to expect this type of barrier, which, for instance, might be explained by a lack of time to attend treatment due to more leisure time activities, such as sports and music, compared to children from lower educated parents [57].

\section{Strengths and limitations}

A strength of our study is that it is the first communitybased evidence regarding parents' and adolescents' expectations of barriers to child and adolescent psychosocial care. Another strength is its extensive recruitment procedure, the successful actions to reduce missing data, and the relatively high response rate.

A limitation of our study might be a potential selection bias. However, we found only small differences between respondents and non-respondents, and between respondents and children in the community, which thus decreases the likelihood of bias. A final limitation is that we did not adjust for correlation of data between adolescents and parents by accounting for the dyadic nature of the data [58, 59]. This is unlikely to have affected our findings in a major way.

\section{Implications}

This study showed that adolescents especially, but also their parents and parents of younger children, expect substantial barriers to psychosocial care, which may greatly 
hinder appropriate care seeking. Barriers are mainly expected regarding treatment, and some groups of parents and adolescents expect barriers more frequently than others do. This evidence may provide support for professionals and policymakers in their attempts to improve access to psychosocial care. For example, intake professionals in treatment settings might address and try to find solutions for barriers among children and their parents who received previous care.

Professionals and policymakers will also, however, need additional evidence concerning the determinants of expectations of barriers, since our study was only able to explain a part of this variation. Major issues pertinent to this might involve the skills that parents and adolescents needed to navigate care as well as personality characteristics and coping styles. Furthermore, it has yet to be confirmed whether expectations of barriers are indeed predictive of actually experiencing barriers later on in the process of entering psychosocial care. Still, a mitigation of these barrier expectations might greatly increase the access to care of those who need it most. Finally, to support specific types of care, insights should be obtained on barriers related to specific types of care or treatment.

Acknowledgments This study has received grants from the Netherlands Organization for Health Research and Development (ZonMw), the Province of Groningen, the University Medical Center Groningen, the University of Groningen, the Menzis health insurance company, and the child and adolescent care providers Elker and Accare.

Conflict of interest On behalf of all authors, the corresponding author states that there is no conflict of interest.

Open Access This article is distributed under the terms of the Creative Commons Attribution 4.0 International License (http://creativecommons.org/licenses/by/4.0/), which permits unrestricted use, distribution, and reproduction in any medium, provided you give appropriate credit to the original author(s) and the source, provide a link to the Creative Commons license, and indicate if changes were made.

\section{References}

1. Briggs-Gowan MJ, Horwitz SM, Schwab-Stone M, Leventhal JM, Leaf PJ (2000) Mental health in pediatric settings: distribution of disorders and factors related to service use. J Am Acad Child Adolesc Psychiatry 39:841-849. doi:10.1097/00004583-200007000-00012

2. Merikangas KR, He JP, Burstein M, Swanson SA, Avenevoli S, Cui L, Benjet C, Georgiades K, Swendsen J (2010) Lifetime prevalence of mental disorders in U.S. adolescents: results from the National Comorbidity Survey Replication-Adolescent Supplement (NCS-A). J Am Acad Child Adolesc Psychiatry 49:980989. doi:10.1016/j.jaac.2010.05.017

3. Patel V, Flisher AJ, Hetrick S, McGorry P (2007) Mental health of young people: a global public-health challenge. Lancet 369:1302-1313. doi:10.1016/S0140-6736(07)60368-7
4. Tick NT, van der Ende J, Verhulst FC (2007) Twenty-year trends in emotional and behavioral problems in Dutch children in a changing society. Acta Psychiatr Scand 116:473-482. doi:10.1111/j.1600-0447.2007.01068.x

5. Zwaanswijk M, Van der Ende J, Verhaak PF, Bensing JM, Verhulst FC (2005) Help-seeking for child psychopathology: pathways to informal and professional services in the Netherlands. J Am Acad Child Adolesc Psychiatry 44:1292-1300. doi:10.1097/01.chi.0000181038.98712.c6

6. Zwaanswijk M, van der Ende J, Verhaak PF, Bensing JM, Verhulst FC (2003) Factors associated with adolescent mental health service need and utilization. J Am Acad Child Adolesc Psychiatry 42:692-700. doi:10.1097/01.CHI.0000046862.56865.B7

7. Zwaanswijk M, van Dijk CE, Verheij RA (2011) Child and adolescent mental health care in Dutch general practice: time trend analyses. BMC Fam Pract 12:133-143. doi:10.1186/1471-2296-12-133

8. Zwaanswijk M, Verhaak PF, Van der Ende J, Bensing JM, Verhulst FC (2006) Change in children's emotional and behavioural problems over a one-year period: associations with parental problem recognition and service use. Eur Child Adolesc Psychiatry 15:127-131. doi:10.1007/s00787-005-0513-4

9. Zwaanswijk M, Verhaak PF, Van der Ende J, Bensing JM, Verhulst FC (2005) Consultation for and identification of child and adolescent psychological problems in Dutch general practice. Fam Pract 22:498-506. doi:10.1093/fampra/cmi045

10. Reijneveld SA, Brugman E, Verhulst FC, Verloove-Vanhorick SP (2004) Identification and management of psychosocial problems among toddlers in Dutch preventive child health care. Arch Pediatr Adolesc Med 158:811-817. doi:10.1001/archpedi.158.8.811

11. Jansen DEMC, Wiegersma P, Ormel J, Verhulst FC, Vollebergh WA, Reijneveld SA (2013) Need for mental health care in adolescents and its determinants: the TRAILS Study. Eur J Public Health 23:236-241. doi:10.1093/eurpub/cks087

12. Brugman E, Reijneveld SA, Verhulst FC, Verloove-Vanhorick SP (2001) Identification and management of psychosocial problems by preventive child health care. Arch Pediatr Adolesc Med 155:462-469. doi:10.1001/archpedi.155.4.462

13. Brannan AM, Heflinger CA, Foster EM (2003) The role of caregiver strain and other family variables in determining children's use of mental health services. J Emot Behav Disord 11:77-91. doi: $10.1177 / 106342660301100202$

14. Morrissey-Kane E, Prinz RJ (1999) Engagement in child and adolescent treatment: the role of parental cognitions and attributions. Clin Child Fam Psychol Rev 2:183-198

15. Owens PL, Hoagwood K, Horwitz SM, Leaf PJ, Poduska JM, Kellam SG, Ialongo NS (2002) Barriers to children's mental health services. J Am Acad Child Adolesc Psychiatry 41:731738. doi:10.1097/00004583-200206000-00013

16. Girio-Herrera E, Owens JS, Langberg JM (2013) Perceived barriers to help-seeking among parents of at-risk kindergarteners in rural communities. J Clin Child Adolesc 42:68-77. doi:10.1080/ 15374416.2012.715365

17. Kazdin AE, Holland L, Crowley M, Breton S (1997) Barriers to treatment participation scale: evaluation and validation in the context of child outpatient treatment. J Child Psychol Psychiatry 38:1051-1062. doi:10.1111/j.1469-7610.1997.tb01621.x

18. Wilson CJ, Deane FP (2012) Brief report: need for autonomy and other perceived barriers relating to adolescents' intentions to seek professional mental health care. J Adolesc 35:233-237. doi:10.1016/j.adolescence.2010.06.011

19. Kodjo CM, Auinger P (2004) Predictors for emotionally distressed adolescents to receive mental health care. J Adolesc Health 35:368-373. doi:10.1016/j.jadohealth.2003.12.005

20. Villagrana M, Palinkas LA (2012) Caregivers' endorsement of barriers to mental health services for children and youth in child 
welfare. J Fam Soc Work 15:111-125. doi:10.1080/10522158.20 12.662672

21. Larson J, Stewart M, Kushner R, Frosch E, Solomon B (2011) Barriers to mental health care for urban, lower income families referred from pediatric primary care. Adm Policy Ment Health 40:159-167. doi:10.1007/s10488-011-0389-1

22. Kazdin AE, Wassell G (1999) Barriers to treatment participation and therapeutic change among children referred for conduct disorder. J Clin Child Psychol 28:160-172. doi:10.1207/ s15374424jccp2802_4

23. Kazdin AE (2000) Perceived barriers to treatment participation and treatment acceptability among antisocial children and their families. J Child Fam Stud 9:157-174

24. Andersen R (1968) A behavioral model of families' use of health services. Center for Health Administration Studies, University of Chicago

25. Andersen RM (1995) Revisiting the behavioral model and access to medical care: does it matter? J Health Soc Behav 36:1-10

26. Andersen RM (2008) National health surveys and the behavioral model of health services use. Med Care 46:647-653. doi:10.1097/MLR.0b013e31817a835d

27. Evenboer KE, Huyghen AMN, Tuinstra J, Reijneveld SA, Knorth EJ (2014) Opening the black box: towards classifying care and treatment for children and adolescents with behavioral and emotional problems (published online ahead of print). Res Soc Work Pract. doi:10.1177/1049731514552049

28. Verhage V, Noordik E, Knorth EJ, Reijneveld SA (2014) Cohort profile: tracing achievements, key processes and efforts in professional care for children and adolescents research; TAKECARE (published online ahead of print). Int J Epidemiol. doi:10.1093/ije/dyu237

29. Reijneveld SA, Wiegersma PA, Ormel J, Verhulst FC, Vollebergh WA, Jansen DE (2014) Adolescents' use of care for behavioral and emotional problems: types, trends, and determinants. PLoS One 9:93526. doi:10.1371/journal.pone.0093526

30. Sayal K (2006) Annotation: pathways to care for children with mental health problems. J Child Psychol Psychiatry 47:649-659. doi:10.1111/j.1469-7610.2005.01543.x

31. Nanninga M, Jansen DEMC, Knorth EJ, Reijneveld SA (2014) Enrolment of children and adolescents in psychosocial care: more likely in low family social support and poor parenting skills. Eur Child Adolesc Psychiatry 24:407-416. doi:10.1007/ s00787-014-0590-3

32. Goodman R (1997) The Strengths and Difficulties Questionnaire: a research note. J Child Psych Psychiatry 38:581-586. doi:10.1111/j.1469-7610.1997.tb01545.x

33. Murphy CE (2005) Parental perceptions of barriers to care: an examination of rural Appalachian parents' expectancies of the availability, process, and outcome of mental health services for elementary school-aged children. Dissertation, Ohio University

34. Guillemin F, Bombardier C, Beaton D (1993) Cross-cultural adaptation of health-related quality of life measures: literature review and proposed guidelines. J Clin Epidemiol 46:14171432. doi:10.1016/0895-4356(93)90142-N

35. Van Widenfelt BM, Goedhart AW, Treffers PD, Goodman R (2003) Dutch version of the Strengths and difficulties questionnaire (SDQ). Eur Child Adolesc Psychiatry 12:281-289. doi:10.1007/s00787-003-0341-3

36. Muris P, Meesters C, van den Berg F (2003) The strengths and difficulties questionnaire (SDQ). Eur Child Adolesc Psychiatry 12:1-8. doi:10.1007/s00787-003-0298-2

37. Crone MR, Vogels AG, Hoekstra F, Treffers PD, Reijneveld SA (2008) A comparison of four scoring methods based on the parent-rated Strengths and Difficulties Questionnaire as used in the Dutch preventive child health care system. BMC Public Health 8:106-114. doi:10.1186/1471-2458-8-106
38. Bouwmans C, Schawo S, Jansen D, Vermeulen K, Reijneveld S, Hakkaart-van Roijen L (2012) Handleiding vragenlijst intensieve jeugdzorg: zorggebruik en productieverlies. (Mannual questionnaire intensive care for youth: health care utilization and productivity loss), Erasmus MC

39. Hakkaart-Van RL (2007) Handleiding TiC-P (Vragenlijst voor zorggebruik en productieverliezen bij psychische aandoeningen). [Manual TiC-P (questionnaire for health care utilization and production loss with psychiatric illness)]

40. Jansen DE, Vermeulen KM, Schuurman-Luinge AH, Knorth EJ, Buskens E, Reijneveld SA (2013) Cost-effectiveness of Multisystemic Therapy for adolescents with antisocial behaviour: study protocol of a randomized controlled trial. BMC Public Health 13:369. doi:10.1186/1471-2458-13-369

41. Centraal Bureau voor de Statistiek (CBS) (2006) Standaard Onderwijsindeling 2006 (Standard Education Classification 2006). http://www.cbs.nl/nl-NL/menu/methoden/classificaties/ overzicht/soi/2006/default.htm. Accessed 27 Sep 2013

42. Achenbach TM, McConaughy SH, Howell CT (1987) Child/ adolescent behavioral and emotional problems: implications of cross-informant correlations for situational specificity. Psychol Bull 101:213. doi:10.1037/0033-2909.101.2.213

43. De Los Reyes A, Kazdin AE (2005) Informant discrepancies in the assessment of childhood psychopathology: a critical review, theoretical framework, and recommendations for further study. Psychol Bull 131:483. doi:10.1037/0033-2909.131.4.483

44. Sourander A, Helstelä L, Helenius H (1999) Parent-adolescent agreement on emotional and behavioral problems. Soc Psychiatry Psychiatr Epidemiol 34:657-663. doi:10.1007/ s001270050189

45. Steinberg L, Silverberg SB (1986) The vicissitudes of autonomy in early adolescence. Child Dev 57:841-851. doi: $10.2307 / 1130361$

46. Frank SJ, Pirsch LA, Wright VC (1990) Late adolescents' perceptions of their relationships with their parents: relationships among deidealization, autonomy, relatedness, and insecurity and implications for adolescent adjustment and ego identity status. J Youth Adolesc 19:571-588. doi:10.1007/BF01537177

47. Raviv A, Raviv A, Vago-Gefen I, Fink AS (2009) The personal service gap: factors affecting adolescents' willingness to seek help. J Adolesc 32:483-499. doi:10.1016/j. adolescence.2008.07.004

48. De Haan AM, Boon AE, Vermeiren RR, de Jong JT (2012) Ethnic differences in utilization of youth mental health care. Ethn Health 17:105-110. doi:10.1080/13557858.2011.645150

49. Cauce AM, Domenech-Rodríguez M, Paradise M, Cochran BN, Shea JM, Srebnik D, Baydar N (2002) Cultural and contextual influences in mental health help seeking: a focus on ethnic minority youth. J Consult Clin Psychol 70:44. doi:10.1037/0022-006X.70.1.44

50. Flink IJE, Beirens TMJ, Butte D, Raat H (2014) Help-seeking behaviour for internalizing problems: perceptions of adolescent girls from different ethnic backgrounds. Ethn Health 19:160 177. doi:10.1080/13557858.2013.801402

51. Paasche-Orlow MK, Wolf MS (2007) The causal pathways linking health literacy to health outcomes. Am J Health Behav 31:S19-S26

52. Churchill R, Allen J, Denman S, Williams D, Fielding K, von Fragstein M (2000) Do the attitudes and beliefs of young teenagers towards general practice influence actual consultation behaviour? Br J Gen Pract 50:953-957

53. Zeijl E, Crone M, Wiefferink K, Keuzenkamp S, Reijneveld M (2005) Kinderen in Nederland [children in the Netherlands]. Sociaal en Cultureel Planbureau, Den Haag

54. De Wolff MS, Vogels AG, Reijneveld SA (2014) The empirical versus DSM-oriented approach of the Child Behavior Checklist: 
similarities and dissimilarities. Eur J Psychol Assess 30:22. doi:10.1027/1015-5759/a000164

55. Eldering L, Knorth EJ (1998) Marginalization of immigrant youth and risk factors in their everyday lives: the European experience. Child Youth Care Forum 27:153-169

56. Crijnen AA, Achenbach TM, Verhulst FC (1997) Comparisons of problems reported by parents of children in 12 cultures: total problems, externalizing, and internalizing. J Am Acad Child Adolesc Psychiatry 36:1269-1277. doi:10.1097/00004583-199709000-00020
57. Bartko WT, Eccles JS (2003) Adolescent participation in structured and unstructured activities: a person-oriented analysis. J Youth Adolesc 32:233-241. doi:10.1023/A:1023056425648

58. Jenkins et al (2009) The use of multilevel modeling for the investigation of family process. Eur J Development Sci 3:131-149. doi:10.3233/DEV-2009-3204

59. McMahon, Pouget ER, Tortu S (2006) A guide for multilevel modeling of dyadic data with binary outcomes using SAS PROC NLMIXED. Comput Stat Data Anal 50:3663-3680. doi:10.1016/j/csda.2005.08.008 